\title{
Electrodeposition of palladium-dotted nickel nanowire networks as a robust self-supported methanol electrooxidation catalyst
}

\author{
Tim Boettcher ${ }^{1, \star}$ (1D, Sasho Stojkovikj ${ }^{2,3}$, Prashant Khadke ${ }^{4}$, Ulrike Kunz ${ }^{1}$, Matthew T. Mayer ${ }^{2}$, \\ Christina Roth ${ }^{4}$, Wolfang Ensinger ${ }^{1}$, and Falk Muench ${ }^{1}$ \\ ${ }^{1}$ Department of Materials and Geoscience, Technische Universität Darmstadt, Alarich-Weiss-Straße 2, 64287 Darmstadt, Germany \\ ${ }^{2}$ Chemical Energy Division, Helmholtz-Zentrum Berlin für Materialien und Energie GmbH, Hahn-Meitner-Platz 1, 14109 Berlin, \\ Germany \\ ${ }^{3}$ Institute of Chemistry and Biochemistry, Freie Universität Berlin, Arnimallee 22, 14195 Berlin, Germany \\ ${ }^{4}$ Faculty of Engineering, Universität Bayreuth, Universitätsstraße 30, 95447 Bayreuth, Germany
}

Received: 26 December 2020

Accepted: 5 April 2021

Published online:

23 April 2021

(C) The Author(s) 2021

\begin{abstract}
Mass activity and long-term stability are two major issues in current fuel cell catalyst designs. While supported catalysts normally suffer from poor long-term stability but show high mass activity, unsupported catalysts tend to perform better in the first point while showing deficits in the latter one. In this study, a facile synthesis route towards self-supported metallic electrocatalyst nanoarchitectures with both aspects in mind is outlined. This procedure consists of a palladium seeding step of ion track-etched polymer templates followed by a nickel electrodeposition and template dissolution. With this strategy, free-standing nickel nanowire networks which contain palladium nanoparticles only in their outer surface are obtained. These networks are tested in anodic half-cell measurements for demonstrating their capability of oxidising methanol in alkaline electrolytes. The results from the electrochemical experiments show that this new catalyst is more tolerant towards high methanol concentrations (up to $5 \mathrm{~mol} \mathrm{~L}^{-1}$ ) than a commercial carbon supported palladium nanoparticle catalyst and provides a much better long-term stability during potential cycling.
\end{abstract}

Handling Editor: N. Ravishankar.

Address correspondence to E-mail: boettcher@ma.tu-darmstadt.de 


\section{GRAPHICAL ABSTRACT}

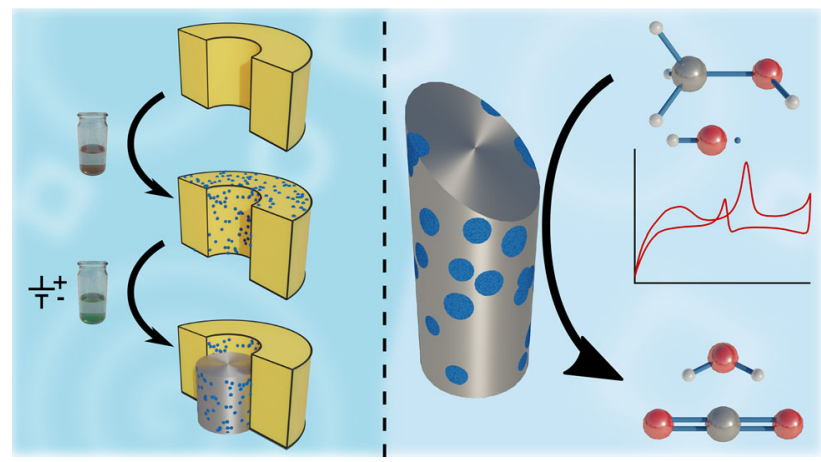

\section{Introduction}

The current industrial standard fuel cell is powered by hydrogen, equipped with a Nafion ${ }^{\mathrm{TM}}$ proton exchange membrane, and platinum nanoparticles on amorphous carbon support as catalyst on anode and cathode side. This setup has been significantly improved over the last decades, [1, 2] but still suffers from fundamental problems that limit broad commercialisation. Among these are poor long-term stability and high cost for platinum and pure hydrogen fuel $[3,4]$. Thus, a lot of research was conducted to optimise or replace many components of the industrial standard fuel cell [5-7].

As an alternative to hydrogen, alcohols such as methanol, ethanol, and ethylene glycol, are investigated as fuels [3]. Since these are all liquids under standard conditions, their transportation, storage, and handling are much easier compared to hydrogen gas. Furthermore, the theoretical energy density of methanol is higher than the one of hydrogen $\left(606 \mathrm{~kJ} \mathrm{~mol}^{-1}\right.$ [8] compared to $70 \mathrm{~kJ} \mathrm{~mol}^{-1}$ [9]). However, the effect of catalyst poisoning by carbonaceous species which heavily decreases performance is much more pronounced for methanol than for hydrogen [10].

The acidic environment of the standard $\mathrm{H}_{2}$ fuel cell strongly limits the number of suitable catalysts to platinum and platinum-based materials [3]. Switching from acidic to alkaline environment allows the application of a broader range of materials, also including less noble materials [3, 11]. Furthermore, in alkaline environment, the kinetics of the oxygen reduction reaction and alcohol oxidation are enhanced, which will boost the overall fuel cell performance [7, 11, 12].

Various catalyst materials were investigated in the last years for numerous reasons, such as to reduce cost of the catalyst material, to improve catalytic activity and/or to reduce poisoning issues. One alternative besides alloying $\mathrm{Pt}$ with elements such as $\mathrm{Ru}$ [6], Sn [13], and Ni [14] is to limit the application to alkaline $\mathrm{pH}$ and switch to another base metal, namely Pd. This metal is also catalytically active for oxygen reduction [15] and-in alkaline regime-for alcohol oxidation [16, 17]. In its pure form it is less active for methanol oxidation than $\mathrm{Pt}$ [16], but much more abundant in the earth crust [10]. Similar to Pt, $\mathrm{Pd}$ can be combined with less noble metals such as $\mathrm{Ni}$ [18]. On the one hand, this reduces the amount of required $\mathrm{Pd}$ for the same amount of catalyst. On the other hand, metals such as $\mathrm{Ni}$ form a thin layer of adsorbed hydroxide ions under alkaline conditions, which helps in alcohol oxidation by providing additional hydroxide species to the catalytically active Pd, which are required for the overall oxidation process [19]. Furthermore, the addition of Ni to Pd slightly alters the electronic structure of $\mathrm{Pd}$ and thus reduces the tendency of catalyst poisoning [20].

Apart from moving from amorphous carbon to more robust supports [2], there are also efforts in manufacturing self-supported catalysts [21]. Since supported catalysts have their catalytically active 
particles attached to the outer support surface, they are prone to dissolution of these particles and thus activity losses [2]. In contrast, the amount of unused catalyst material is very low due to the high surfaceto-volume ratio [22]. Self-supported catalysts circumvent the problem of carbon corrosion and tend to be less prone to degradation mechanisms such as Ostwald ripening, particle detachment, and particle agglomeration, but have to make a compromise between mechanical stability and mass activity [21].

One example for this trade-off is the synthesis of one-dimensional (1D) nanostructure catalysts. Using template-based approaches, a large variety of structural diversity can be achieved by tuning the template manufacturing process or the synthesis itself [21]. For example, ion track-etched polymer templates, which were irradiated with a parallelised ion beam, can be used to manufacture parallelly aligned individual nanowires by means of electrodeposition [20]. The mass activity of these is directly related to their surface-to-volume ratio, which depends on the aspect ratio. With an increasing aspect ratio, i.e. increasing the length and reducing the diameter of nanowires, the mass activity can be enhanced. However, these nanowires tend to cluster during the removal of the polymer template the higher the aspect ratio becomes [23]. By creating intersections between the single template pores by performing multiple parallel irradiations at different angles [24] or by simple defocusing of the ion beam, crossing ion tracks are formed, which lead to crossing pores inside the polymer template. Thus, a nanowire network where individual nanowires intersect with each other and provide a self-supported structure is formed. Apart from improved mechanical stability, such nanowire networks tend to improve diffusion paths inside the catalyst layer and can be seen as a promising candidate for support structures [10, 25].

In this work, a synthesis route for the fabrication of three-dimensional nanowire networks with catalytically active particles embedded in their outer surface is presented. The synthesis starts with the seeding of a polymer template by means of swelling activation followed by the growth of the nanowire network inside the template using electrodeposition. This approach can be applied to various material combinations and is promising for achieving high mass activities, while not being prone to catalyst detachment, since the catalyst is not attached to the support surface but embedded into it. The synthesis route is illustrated in detail, and the obtained catalyst is characterised with respect to its morphology and composition. Electrochemical applicability is demonstrated by performing the oxidation of methanol in alkaline electrolyte in half-cell measurements.

\section{Materials and methods}

\section{Chemicals}

All solutions were freshly prepared and all glassware was cleaned with boiling aqua regia prior to use. For the preparation of all solutions, purified water (Milli$Q,>18.2 \mathrm{M} \Omega \mathrm{cm}$ ) and the following chemicals were used without further purification: borane dimethylamine complex (Sigma-Aldrich, 97\%), boric acid (Sigma-Aldrich, > 99.5\%), (DCM) (Merck, > 99.8\%), ethanol (Roth, > 99.8\%), hydrochloric acid (37\%, AppliChem, for analysis; 32\%, J.T. Baker, Baker Analyzed purity grade), methanol (AppliChem, > 99.5\%), Nafion solution (5wt.\%, EC-NS-05, ElectroChem Inc.), nickel(II) chloride (Aldrich, 98\%), nitric acid ( 70\%, Sigma-Aldrich, > 99.999\%), palladium on carbon (10 wt.\%, activated carbon support, Aldrich), palladium(II) chloride (Alfa Aesar, 99.9\% metal basis), sodium hydroxide (Grüssing, > 99\%).

\section{Nanostructure synthesis}

Commercially available ipPORE polycarbonate membranes from it4ip with a thickness of $25 \mu \mathrm{m}$, an ion fluence of $1.5 \times 10^{8} \mathrm{~cm}^{-2}$, and pore diameters of $400 \mathrm{~nm}$ were first activated by means of swelling activation [26]. Therefore, the membranes were immersed in a solution of $0.5 \mathrm{M}$ borane dimethylamine complex (DMAB) in methanol for $2 \mathrm{~min}$ (swelling step). Afterwards, they were rinsed with water two times and transferred to a solution of $59 \mathrm{mM} \mathrm{PdCl}_{2}$ and $108 \mathrm{mM} \mathrm{HCl}$ in water for another 2 min (activation step) followed by another rinsing step in water. This procedure of swelling and activation was repeated up to six times to achieve different Pd loadings. After this nanoparticle deposition process, the template surface was gently rubbed with an ethanol-containing tissue to remove Pd nuclei, which have formed on the outer template surfaces.

For the electrodeposition of $\mathrm{Ni}$, a Ni back electrode was sputtered onto one surface of the membranes 
using a Quorum Q300T D sputter coater. The sputter current was adjusted to $100 \mathrm{~mA}$ and applied for $120 \mathrm{~s}$ to form a conductive layer. Electrodeposition was carried out at a potential of $-1.0 \mathrm{~V}$ vs. $\mathrm{Ag} \mid \mathrm{AgCl}$ (Meinsberg, in $3 \mathrm{M} \mathrm{KCl},+210 \mathrm{mV}$ vs. SHE) in an electrolyte composed of $0.5 \mathrm{M} \mathrm{NiCl}_{2}$ and $0.5 \mathrm{M} \mathrm{H}_{3} \mathrm{BO}_{3}$ in purified water. The deposition was stopped when the current started to rapidly increase after the template pores were completely filled. After electrodeposition, the $\mathrm{Ni}$ back electrode was removed by dabbing and rubbing the surface with a cotton bud impregnated with aqua regia so that only the polymer template with the nanowire network inside remained.

\section{Characterisation}

For scanning electron microscopy (SEM), samples were immersed in fresh DCM three times for $30 \mathrm{~min}$ each to remove the polymer template. Afterwards, the plain nanostructures were glued to an SEM sample holder with Planocarbon N650 carbon glue to ensure electric conductivity. SEM measurements were performed on a Philips XL30 FEG using an accelerating voltage of $25 \mathrm{kV}$. For energy-dispersive X-ray spectroscopy (EDX) measurements, an EDAX CDU LEAP detector was used.

For the transmission electron microscopy (TEM) measurements, a small piece of each sample (still located in the polymer template) was embedded in Araldite ${ }^{\circledR} 502$ resin. This resin was cured at $60^{\circ} \mathrm{C}$ for 16h. Afterwards, the obtained resin block was cut into thin slices of approximately $70 \mathrm{~nm}$ thickness by a Reichert-Jung Ultracut E ultramicrotome equipped with a diamond knife by Delaware Diamond Knives. The cut slices were collected on a $\mathrm{Cu}$ grid and then investigated with a FEI CM20 operating at $200 \mathrm{kV}$. EDX measurements were performed using an Oxford X-Max detector.

The precise amounts of $\mathrm{Ni}$ and $\mathrm{Pd}$ were determined by inductively coupled plasma optical emission spectroscopy (ICP-OES). Analyses were conducted on an iCAP 7400 Duo MFC ICP-OES Analyzer by Thermo Scientific. After dissolving the polymer template, the samples were dissolved in $4.5 \mathrm{~mL}$ aqua regia diluted with $5 \mathrm{~mL}$ of $\mathrm{H}_{2} \mathrm{O}$. This solution was then further diluted with $3 \mathrm{wt} . \% \mathrm{HNO}_{3}$ prior to the measurements.

Electrochemical measurements were carried out on a Gamry Reference $600+$ potentiostat. Prior to the measurements, the polymer template was completely dissolved by repeatedly rinsing the samples with DCM similar to SEM preparation. The remaining nanowire network was then weighed on a balance, placed on a glass slide, and electrically contacted by a $\mathrm{Ni}$ wire with $0.5 \mathrm{~mm}$ diameter in the measurement cell. For the reference catalyst, $15.64 \mathrm{mg}$ of Pd@C powder and $50 \mathrm{~mL}$ of $5 \mathrm{wt} . \%$ Nafion $^{\mathrm{TM}}$ solution were mixed with $1 \mathrm{~mL}$ of water. This suspension was then ultrasonicated for approximately $10 \mathrm{~min}$. Subsequently, $2.5 \mu \mathrm{L}$ of the suspension (containing $3.5 \mu \mathrm{g}$ of Pd) was deposited on the glassy carbon electrode and left to dry in air for $15 \mathrm{~min}$. Both preparation techniques are also schematically illustrated in the Supporting Information, Figs. S1 and S2.

The so prepared electrodes were then connected to the potentiostat as working electrode. A platinised Ti mesh was used as a counter electrode and an $\mathrm{Ag} \mid \mathrm{AgCl}$ electrode (Meinsberg, in $3 \mathrm{M} \mathrm{KCl},+210 \mathrm{mV}$ vs. SHE) as reference electrode, respectively. All measurements were carried out in unstirred $0.1 \mathrm{M}$ $\mathrm{NaOH}$ solution. For electrocatalytic measurements, methanol was added in various concentrations. The scan range was set to $-1.0 \mathrm{~V}$ to $0.5 \mathrm{~V}$ while the scan rate was adjusted to $50 \mathrm{mV} \mathrm{s}^{-1}$. Also, automated iR drop-correction via current interrupt technique was applied to all measurements [27, 28]. For the longterm measurements, the alcohol concentration was fixed at $0.25 \mathrm{M}$ and the scan rate was increased to $100 \mathrm{mV} \mathrm{s}^{-1}$.

\section{Results and discussion}

A schematic drawing of the synthesis route of nanoparticle-decorated (dotted) nanowires is shown in Fig. 1. The aim of this route is to combine surfaceselective chemical reduction of nanoparticles, i.e. the seeding process to obtain nanoparticles on the pore wall, and electrodeposition, i.e. a complete filling of the template pores, to create a robust catalyst material with efficient noble metal utilisation by providing catalytically active particles only embedded in the outer catalyst surface.

The first step of nanowire fabrication is the socalled swelling activation [26, 29]. Here, Pdnanoparticles which adhere to the template pore walls are created. The aim of this step is to generate a large amount of small particles, which are well-dispersed on the polymer template surface, to obtain a 
high amount of Pd-surface area. Swelling activation was used instead of conventional Sn-Pd activation to avoid contamination by $\mathrm{Sn}$ species [30].

In swelling activation, the polymer template is first immersed in a swelling solution. For polycarbonate templates, this solution is based on methanol which is absorbed by the polymer membrane and leads to a swelling of the material, i.e. a less dense packing of the single polycarbonate chains [31]. This widening enables the absorption of the reducing agent DMAB into the template material. Afterwards, the template is rinsed with water to remove any non-absorbed methanol and DMAB species, which could lead to homogeneous nucleation of $\mathrm{Pd}$ particles inside the template pores, and subsequently placed in the activation solution that contains Pd ions. These metal ions react with the reducing agent at the polymerliquid interface and form small nanoparticles that adhere to the polymer surface while the reducing agent is oxidised. As a result of this activation

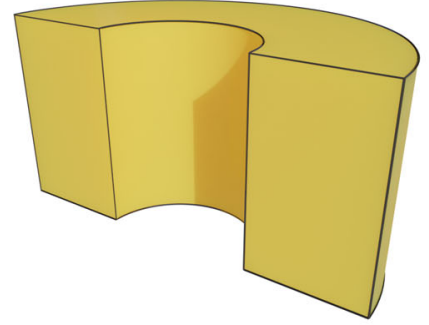

(a)



(e)



(b)



(f)



Figure 2 Photographs of the six times activated sample in different stages of the synthesis. The left image shows the template prior to the synthesis. In the middle image, the template was seeded with Pd nuclei leading to a brownish tint. The right image shows the final circular Pd-dotted Ni nanowire network after electrodeposition and subsequent dissolution of the polymer template.

procedure, the polymer template changes colour from translucent white to translucent brownish, as indicated in Fig. 2. The brownish tint is caused by the presence of $\mathrm{Pd}$ nanoparticles, and thus indicates a successful reduction procedure. These steps can be repeated to increase both amount and size of deposited nanoparticles which leads to a stronger tint

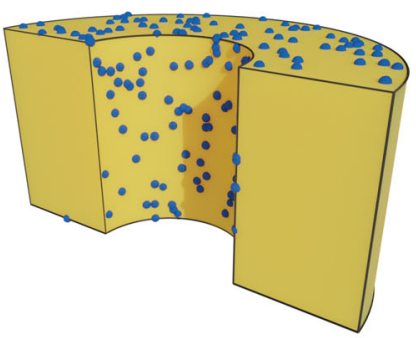

(c)

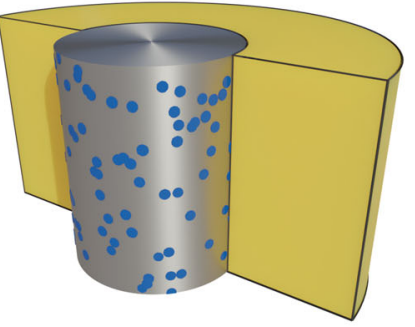

(g)

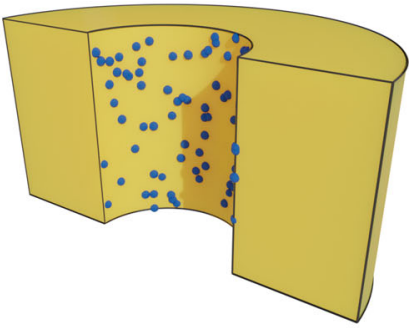

(d)

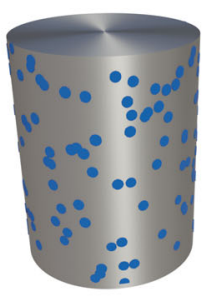

(h)
Figure 1 Synthesis route of dotted nanowires. The (a) etched polymer template is (b) soaked with reducing agent by immersing it in the swelling solution. Afterwards, it is transferred to the activation solution, where (c) Pd (blue dots) form on the template surface by a reaction between the reducing agent and the metal precursor. (d) After removing the noble metal seeds on the outer template surfaces, (e) a back electrode of $\mathrm{Ni}$ (indicated by the arrow) is applied via sputtering. (f) Then, template pores are filled with Ni via electrodeposition. The Pd seeds are thus embedded in the outer nanostructure surface. Finally, (g) the back electrode and (h) the template are removed so that the plain nanostructure remains. For the sake of simplicity, the process is illustrated on a single nanowire but can also be employed in many template pores at the same time to form network structures. 
of the polymer membrane. In this study, polymer templates were activated two, four, and six times to obtain nanostructures with various noble metal loadings.

In the subsequent electrodeposition step, $\mathrm{Ni}$ nanowires are grown along the template pores starting from the back electrode of the polymer template through the membrane to the other side. Growth of the structures is initiated at an electrode that is applied to one side of the template by sputtering. Since the growing nanostructures are connected to the back electrode, these act as extensions of the electrode into the template pores. This also explains why it is extremely important that the $\mathrm{Pd}$ nuclei are separated from each other and do not form a conductive chain through the polymer template. A continuous conductive path through the pores would lead to voids in the final structure and early growth of caps and top layer on top of the polymer template. This might shield other pores from the electrolyte leading to inhomogeneous wire growth. A schematic drawing for this is provided in the Supporting Information, Fig. S3. However, for the employed nanoparticle loadings structure growth was not disturbed by any unintended continuous conductive paths along the polymer template, as shown later.

After electrodeposition, the polymer template is dissolved and a free-standing network of intersecting $\mathrm{Ni}$ nanowires with Pd nanoparticles embedded in the outer surface is obtained. The network structure is caused by using a commercial filter membrane as template which does not require a parallelised ion beam. The obtained network can easily be handled by an ordinary tweezer as shown in the right image of Fig. 2.

It was also possible to manufacture $\mathrm{Ni}$ nanowire networks decorated with embedded Ag particles (see Supporting Information, Figs. S4, S5). These structures could act as a catalyst for oxygen reduction reaction in alkaline $\mathrm{pH}$ [32]. We believe that this synthesis route can be expanded to many more material combinations as long as the deposited nanoparticles are more noble than the nanowire network material. Otherwise, galvanic exchange would become a competing process that would dissolve the deposited nanoparticles, while the template pores are filled with nanowires.

\section{SEM}

Figure 3a shows SEM images of the fabricated nanowire networks. Although the single nanowires are stochastically aligned, the mechanical stability of the network is remarkably high, as seen in Fig. 2. The height of the network amounts to approximately $25 \mu \mathrm{m}$. In combination with the lack of cap growth, i.e. $\mathrm{Ni}$ deposition occurring on top of the polymer membrane after completely filling the template pores, this indicates a good control over the electrodeposition process with homogeneous wire growth along the whole sample. The back electrode removal by careful dabbing with aqua regia also proves to be a precise process, since no remains of the back electrode are visible, while no damage caused by etching could be observed on the edges of the obtained nanowires. The detailed view shown in the inset of Fig. 3a indicates continuous and smooth-walled nanowires. This suggests a good integration of the Pd nanoparticles into the nanowire surface. Furthermore, since no voids in nanowire growth can be observed, it can be deduced that the density of Pd seeds was low enough and their size sufficiently small to not provide any extended conductive paths and thus ensure void-free nanowire growth along the template pores. Similar results were obtained for the two and four times activated samples. An EDX spectrum of a nanowire network that was activated six times is shown in Fig. $3 \mathrm{~b}$. The Pd signal is very low as expected and corresponds to approximately 4.9 wt.\% of Pd when quantifying only Ni and Pd. For samples with fewer activation steps, the Pd signal becomes even weaker and can no longer be distinguished from the background spectrum.

\section{TEM}

To obtain information about the spatial distribution and nanostructure of the Pd particles on the six times activated sample, TEM was performed. An image of a cross section of one single nanowire is shown in Fig. 4a. Due to the disordered alignment of the single nanowires, it cannot be deduced, whether this cross section was deformed during the TEM preparation or whether the nanowire was not aligned orthogonally to the cutting plane. From the image itself it can be observed that there are no voids inside the nanowire. Also, there is no clear visual indication of $\mathrm{Pd}$ 
nanoparticles which are embedded in the outer nanowire surface.

TEM EDX was performed on the two spots marked in Fig. 4a. The corresponding spectra are shown in Fig. 4b. The spectrum that was recorded in the centre of the nanowire shows only signals for $\mathrm{Ni}, \mathrm{Cu}$, and $\mathrm{Si}$. Since the $\mathrm{Cu}$ and $\mathrm{Si}$ signals are caused by the TEM grid which held the sample slices and the surrounding TEM equipment, respectively, it can be stated that the centre of the nanowire consists only of pure $\mathrm{Ni}$. The spectrum recorded in the outer parts of the nanowire shows additional signals for $\mathrm{C}, \mathrm{O}$, and $\mathrm{Pd}$. While it is very likely that the signals for $\mathrm{C}$ and $\mathrm{O}$ can be attributed to the surrounding polymer resin and polymer template, the Pd signals are caused by the particles that were formed in the swelling activation process. Considering only the $\mathrm{Ni}$ and $\mathrm{Pd}$ signal, a quantification of this local EDX signal yields approximately $0.85 \mathrm{wt} . \% \mathrm{Pd}$ and $99.15 \mathrm{wt} . \% \mathrm{Ni}$. This result verifies the successful implantation of $\mathrm{Pd}$ in the outer surface of the Ni nanowire network. To further fortify this, a TEM mapping was performed on another cross section of the same sample (see Supporting information, Fig. S6), which again shows the presence of Pd on the edge of the cross section.

\section{ICP-OES}

ICP-OES measurements were performed on the two, four, and six times activated samples. The obtained fractions of $\mathrm{Ni}$ and $\mathrm{Pd}$ in wt.\% are shown in Table 1. The values for $\mathrm{Pd}$ are very low (in the range of $0.6 w t . \%$ to $2.4 w t . \%$ ).

\section{Electrochemical characterisation}

In Fig. 5, the iR drop-corrected cyclic voltammetry (CV) curves for two, four, and six times activated dotted samples in $0.1 \mathrm{M} \mathrm{NaOH}$ solution are depicted. Furthermore, an iR drop-corrected CV curve of the prepared commercial Pd@C reference catalyst is shown. Data normalisation was performed by dividing the measured currents by the mass of $\mathrm{Pd}$ contained in each sample as derived from the sample weight and ICP-OES measurements.

A total of six different peaks can be observed for the dotted structures. The peak at $-706 \mathrm{mV}$ for the two times activated sample, $-596 \mathrm{mV}$ for the four times activated sample, and $-745 \mathrm{mV},-637 \mathrm{mV}$ and $-556 \mathrm{mV}$ for the six times activated sample (peaks I-

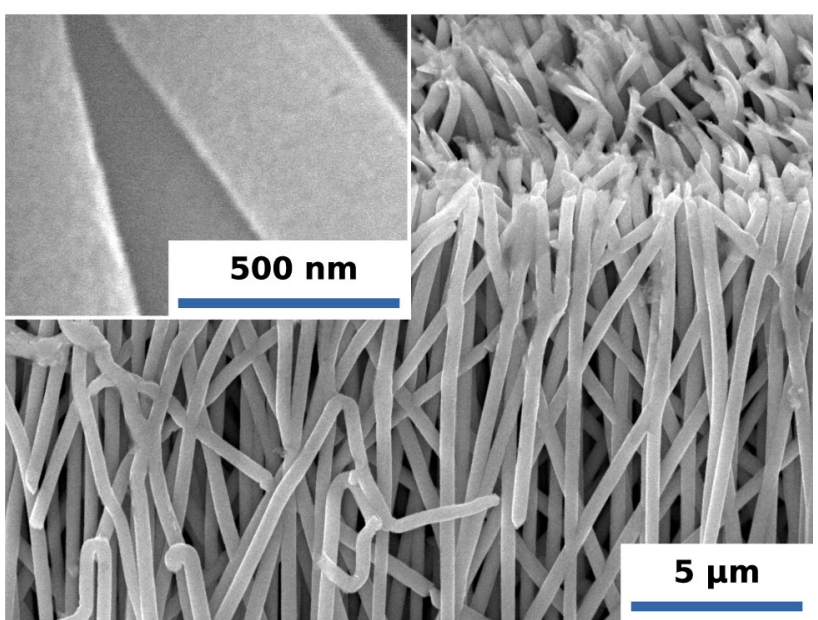

(a)

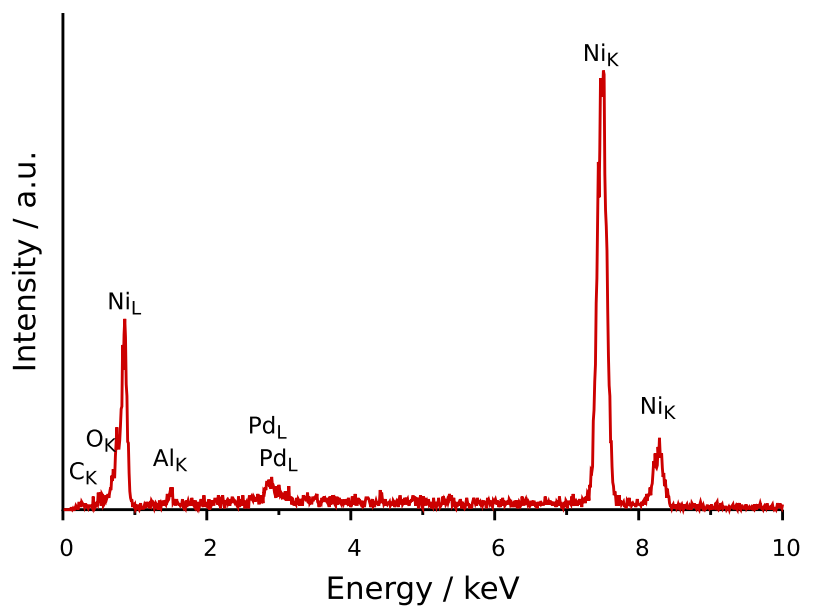

(b)

Figure 3 (a) SEM images of the obtained nanowire networks, in this case a six times activated sample. Wires have grown continuously and do not show any voids. Furthermore, the outer nanowire wall is smooth and does not give any hints on the presence of Pd seeds. (b) EDX spectrum of the nanowire network shown in Fig. 3a. Most of the sample consists of $\mathrm{Ni}$, while only approximately $4.9 \mathrm{wt}$. \% consist of Pd. The observed aluminium peak is caused by the SEM sample holder which was in close vicinity to the shown sample region.

III) can be associated with the desorption of hydrogen species and the adsorption of oxidic/hydroxidic species at the Pd surface $[17,33,34]$. In the range from $+426 \mathrm{mV}$ to $+460 \mathrm{mV}$, a reduction peak which can be associated with the reduction of $\mathrm{NiOOH}$ species to $\mathrm{Ni}(\mathrm{OH})_{2}$ (peak IV) is found $[17,35,36]$. The fifth peak (peak V) that can be identified occurs in the range from $-250 \mathrm{mV}$ to $-228 \mathrm{mV}$ and is commonly attributed to the reduction of $\mathrm{PdO}$ species to $\mathrm{Pd}$ $[34,37,38]$. The corresponding oxidation peak of $\mathrm{Pd}$, 
Table 1 Mass fractions in wt.\% of samples with various amounts of activation steps as determined by ICP-OES

\begin{tabular}{lll}
\hline Steps & Ni / wt. $\%$ & Pd / wt.\% \\
\hline 2 & 99.41 & 0.59 \\
4 & 98.32 & 1.68 \\
6 & 97.65 & 2.35 \\
\hline
\end{tabular}

which can be found in anodic scan direction at values around $0 \mathrm{mV}$ according to literature, is not observed in this case [34, 37]. At approximately $-640 \mathrm{mV}$ (peak VI) the adsorption of hydrogen species occurs [34].

When comparing this to the $\mathrm{Pd@C}$ reference catalyst, it can be seen that the reference catalyst shows two more peaks in close vicinity to the Pd reduction peak in both anodic $(-264 \mathrm{mV})$ and cathodic $(-326 \mathrm{mV})$ scan direction (marked with an asterisk). It remains unclear to which chemical reactions these peaks correspond to. However, similar peaks are found for Pd@C catalysts in the literature [39].

The same set of samples was then exposed to various concentrations of methanol and again measured by means of $\mathrm{CV}$. The obtained curves for a methanol concentration of $0.25 \mathrm{~mol} \mathrm{~L}^{-1}$ are shown in Fig. 6. At first glance, two new peaks can be noticed: one is located at approximately $-40 \mathrm{mV}$ in anodic scan direction, the other one is found between $-214 \mathrm{mV}$ and $-230 \mathrm{mV}$ in cathodic scan direction. Both peaks can be associated with the oxidation of methanol species at the Pd surface [34, 37]. Further investigation of the curves reveals an attenuation of the $\mathrm{H}$ desorption and $\mathrm{OH}^{-}$adsorption peaks and the vanishing of the $\mathrm{H}$ adsorption peak.

Both the vanishing of the $\mathrm{H}$ adsorption peak and the attenuation of the other two peaks can be explained by the presence of methanol in the alkaline electrolyte, since methanol also adsorbs on the Pd surface and thus reduces the number of available sites for other ad-/desorption reactions [33]. The same attenuation can also be observed for the Pd@C reference catalyst.

One feature that is unique to the Pd@C reference catalyst is two loops in the alcohol CV, both in close vicinity to the alcohol oxidation reaction peaks. These loops are caused by the automatic iR drop correction during the measurements, which was performed using the current interrupt technique. By adjusting the applied voltage from one data point to the next, this technique stretches and compresses the $\mathrm{x}$-axis of the cyclovoltammograms depending on the voltage drop of the previous data point, which itself is depending on the measured electrical current [27]. For rapid changes in current followed by a slower current change, this can result in a strong deformation of the $x$-axis followed by its relaxation. This overshooting of the artificial control circuit imposed by the current interrupt technique thus leads to the formation of measurement loops.

For all samples discussed so far, CV measurements were performed with further concentrations of methanol. This is exemplarily shown in Fig. 7 for the

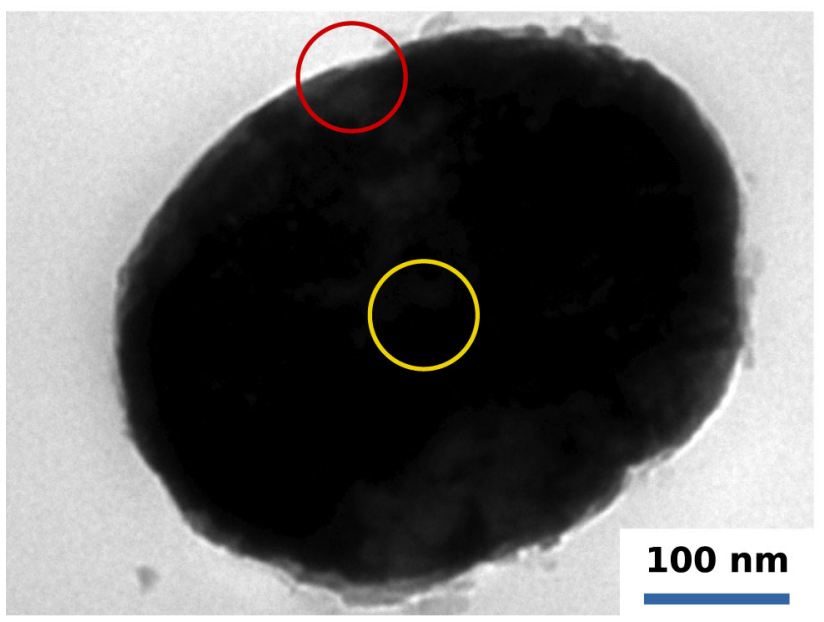

(a)



(b)

Figure 4 (a) TEM image of the cross section of a single Pddotted Ni nanowire embedded in polymer resin. The two circles indicate locations where EDX was performed. (b) EDX spectra of the two spots marked in Fig. 4a. The outer part of the cross section shows a clear but weak Pd signal, while the centre of the cross section consists of pure Ni. 
two times activated sample. For the other curves, the reader is referred to the Supporting Information, Figs. S7-S9. For the dotted samples, the two peaks associated with the methanol oxidation reaction continuously increase in intensity with increasing methanol concentration, while the peaks associated with $\mathrm{H}$ ad-/desorption and $\mathrm{OH}^{-}$adsorption become attenuated. This fortifies the aforementioned correlation between the concentration of methanol in the electrolyte and the intensity of $\mathrm{OH}^{-}$adsorption due to the limited amount of available Pd catalyst sites.

A comparison of the mass activities (i.e. the normalised peak currents) of the dotted samples and the reference catalyst indicates higher mass activities for the latter one for alcohol concentrations up to $1.00 \mathrm{M}$ (see Fig. 8). Since the obtained currents were normalised to the mass of the Pd content of the samples, this indicates a higher amount of exposed surface area for the reference catalyst. On the one hand, this can be caused by a higher surface-to-volume ratio of the Pd nanoparticles of the reference catalyst. Since the surface-to-volume ratio of spherical particles decreases with $r^{-1}$ with increasing radii $r$ and thus also activity decreases $[2,37]$, this would indicate that the reference catalyst contains smaller catalyst particles than the dotted samples. On the other hand, the dotted structures have their catalytic particles not attached to the substrate surface but embedded into it, which leads to a decrease in exposed area for catalytic nanoparticles of identical dimensions.

Thus, a high-surface-to-volume ratio and a high amount of small Pd nanoparticles become even more important.

The latter was tried to be achieved by increasing the amount of activation cycles. In Fig. 9, various possibilities of nanoparticle growth during activation are shown schematically. While the first scheme column shows well separated nanoparticles with roughly the same dimensions, the second column shows an agglomeration of small nanoparticles and the third column shows the growth of one large nanoparticle instead of nucleation and formation of multiple nanoparticles. When comparing these three possibilities with regard to the expected mass-activities of the exposed catalyst, the mass activity would stay constant for the first schematic and growing numbers of nanoparticles. Only the absolute currents would increase. For the second and third case, mass



Figure 5 iR drop-corrected CV curves of two, four, and six times activated Pd-dotted Ni nanowire networks as well as the Pd@C reference catalyst in $0.1 \mathrm{M} \mathrm{NaOH}$ solution at a scan rate of $50 \mathrm{mV} \mathrm{s}^{-1}$. The arrows indicate the scan direction.

activities would decrease with an increasing amount of deposited material.

However, mass activities increase with an increasing number of activation treatments, as derived from Fig. 6. This mismatch can either be attributed to compositional differences between the samples used in ICP-OES and the samples applied in half cell measurements or a not yet discovered parameter during swelling activation. Although compositional differences cannot be completely excluded, these also boil down to some experimental variance. We believe that the rinsing step between the swelling step and the activation step during nanoparticle deposition might cause these variances. When rinsing the sample between the two steps, there is a conflict of rinsing for too long, i.e. allowing too much of the absorbed reducing agent to leave the polymer again, and rinsing too short, i.e. not properly cleaning the template pores. While the first option would lead to lower amounts of reducing agent available for metal reduction, the latter option can lead to remains of the swelling solution inside the template pores and thus an increased amount of reducing agent for metal reduction above the intended value. Also, this might lead to nucleation inside the template pores followed by sedimentation of the so-formed nanoparticles at the pore walls. This would then lead to a difference in composition although the same procedure was carried out and 
thus would have an impact on the applied data normalisation.

When looking at the CV curves of the dotted structures in comparison to the Pd@C reference catalyst in general, it becomes obvious that the latter one provides higher mass activities than each of the dotted structures for alcohol concentrations up to $1.00 \mathrm{M}$. At higher concentrations, the measured mass activity of the reference catalyst dramatically decreases and is then surpassed by all dotted structures. The increased mass activities for low alcohol concentrations of the reference catalyst can be explained by the exposed surface of the $\mathrm{Pd}$ nanoparticles for both catalyst types and the applied normalisation. The dotted structures, however, prove to be more tolerant to high alcohol concentrations as indicated by the measured mass activities at alcohol concentrations of $5.00 \mathrm{M}$. One possible reason for this might be a synergistic effect between the Pd catalyst and the less noble $\mathrm{Ni}$, where the catalyst is responsible for oxidising methanol, while the surrounding $\mathrm{Ni}$ substrate can provide additional $\mathrm{OH}^{-}$ions for facilitated oxidation of methanol, even at high concentrations of alcohol [19]. This is supported by An et al. who report an activity loss caused by a high concentration of ethanol and thus blocking of the active sites by adsorbed alcohol species [11].

To further investigate the performance of the dotted nanowire networks, long-term cycling in the same measurement range as before and with the

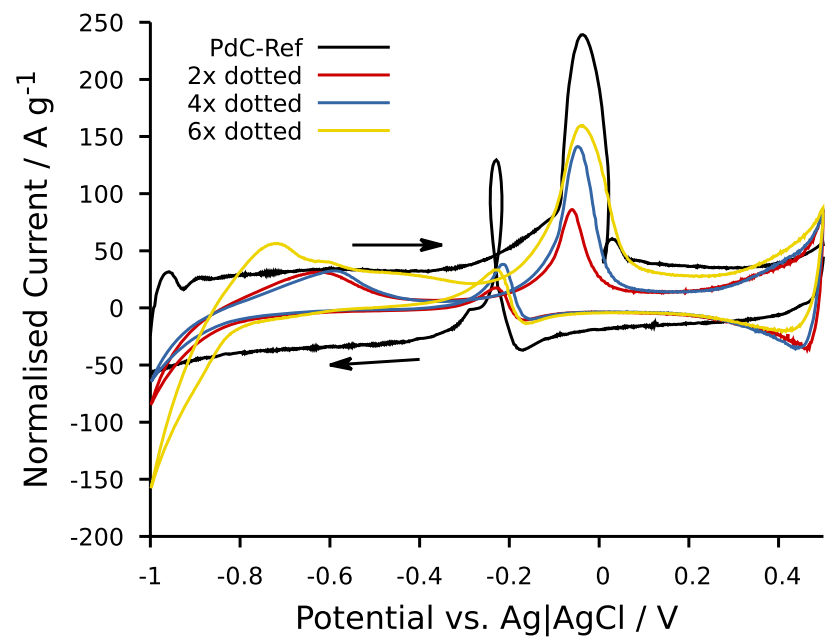

Figure 6 iR drop-corrected CV curves of two, four, and six times activated Pd-dotted $\mathrm{Ni}$ nanowire networks in $0.1 \mathrm{M} \mathrm{NaOH}$ solution containing $0.25 \mathrm{M}$ methanol at a scan rate of $50 \mathrm{mV} \mathrm{s}^{-1}$. The arrows indicate the scan direction. addition of $0.25 \mathrm{M}$ methanol to the electrolyte was performed at a scan rate of $100 \mathrm{mV} \mathrm{s}^{-1}$ after the aforementioned concentration studies. The obtained results are compared against the commercial Pd@C reference catalyst and illustrated in Fig. 10. For evaluation, the charge correlated with the methanol oxidation peak in anodic scan direction was determined. Normalisation was then performed with respect to the maximum charge obtained during the whole measurement.

The Pd@C reference catalyst shows an exponential decrease in activity over the whole measurement. Only approximately $4 \%$ of the maximum charge remain after 1000 cycles. The two and four times activated dotted structures behave differently in the first 100 measurement cycles, but then show a much weaker decay from $100 \%$ to $92 \%$ of the maximum charge and from $84 \%$ to $79 \%$ of the maximum charge, respectively. Interestingly, the six times activated sample does neither show a decrease in performance, nor a constant value, but indicates an increase in performance over time. Given the possibility of methanol evaporation during the measurement and thus a decrease of fuel concentration in the analyte solution, this is even more remarkable. However, due to the numerous factors which may influence longterm stability testing and the fact that an investigation of these would already be an own study, the reasons for this behaviour shall not be discussed here. Instead, it shall be pointed out, that the overall



Figure 7 iR drop-corrected $\mathrm{CV}$ curves of a two times activated Pd-dotted Ni nanowire network in $0.1 \mathrm{M} \mathrm{NaOH}$ solution with various methanol concentrations at a scan rate of $50 \mathrm{mV} \mathrm{s}^{-1}$. The arrows indicate the scan direction. 


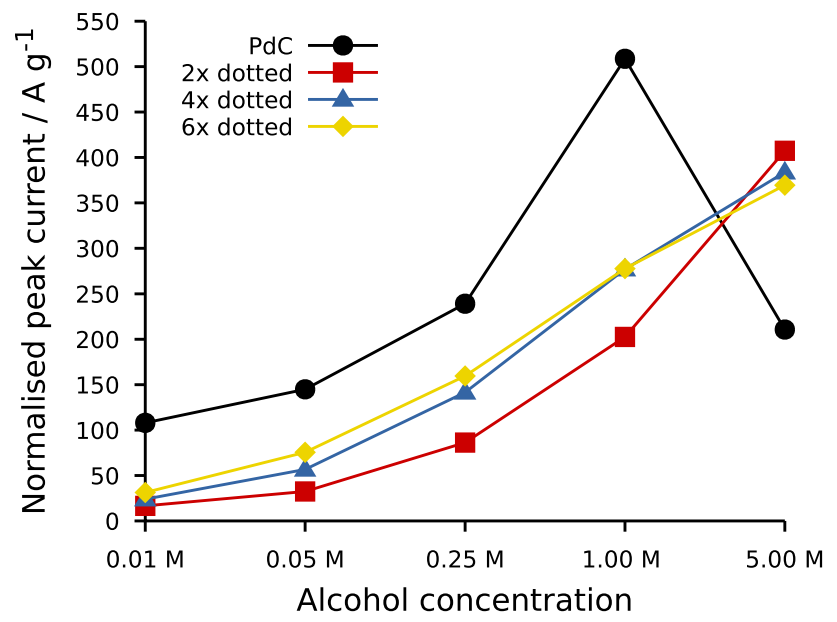

Figure 8 Normalised peak currents of the alcohol oxidation peak in anodic scan direction for the two, four, and six times activated dotted samples as well as the commercial Pd@C reference catalyst.

long-term stability of the dotted nanostructure catalysts is much higher than the one of the Pd@C reference catalyst.

To further examine the long-term stability of both the dotted nanowire networks and the Pd@C reference catalyst, SEM was carried out on each sample before and after the long-term stability test. The dotted samples did not show any change in morphology (see Supporting Information, Fig. S10). Their appearance was the same as shown in Fig. 3a. However, the Pd@C reference catalyst significantly
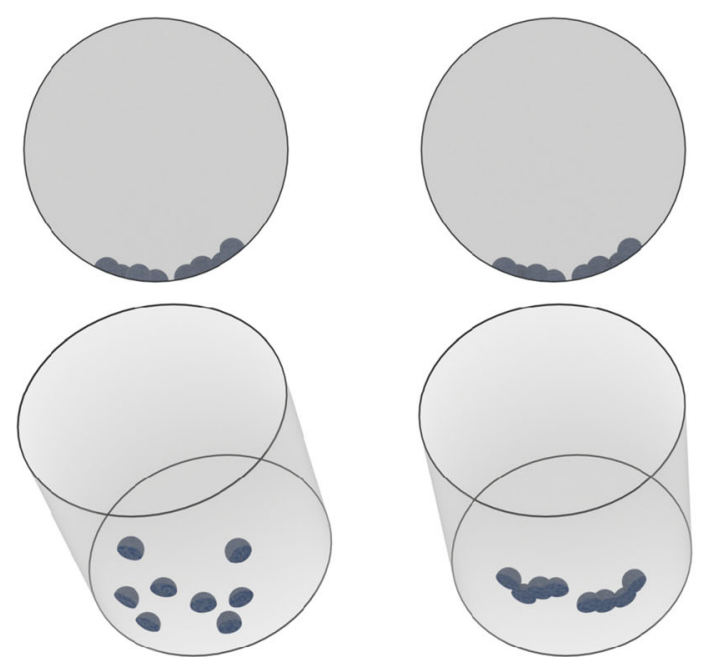

Figure 9 Schematic illustration of various nanoparticle alignments in the nanowire after swelling activation and electrodeposition. Apart from the formation of individual nanoparticles during activation (first column), it is also possible for agglomerates to form at the pore surface (second column) or for changed its shape during the test, as shown in Fig. 11. The image on the left shows a dried droplet of the reference catalyst suspension before the test. Here, single platelets of carbon decorated with $\mathrm{Pd}$ can be observed and clearly distinguished from each other. After the test, the single platelets can hardly be discriminated. Instead, they changed their morphology and formed one large agglomerate with probably decreased surface area. This might explain the drop in performance of the reference catalyst observed in the long-term stability test (see Fig. 10).

Also, the electrolytes of the long-term cycling tests were investigated by means of ICP-OES to determine any metal losses during the cycling test and to further evaluate the possible degradation mechanisms of the catalyst. For all dotted samples, no Pd could be detected in the electrolytes. Thus, particle detachment and dissolution which are both severe degradation mechanisms for noble metal nanoparticlebased catalysts do not contribute to the change of the performance of the dotted catalyst. Also, Ni species could not be detected, which indicates that the nanowire network did not corrode, either. The same analysis was then performed on the electrolyte of the Pd@C reference catalyst. Here, no Pd could be detected in the used electrolyte. From this, it can be concluded that the decrease in performance of the Pd@C reference catalyst cannot be caused by catalyst particle detachment, or particle dissolution but must



single nanoparticles to increase in size (third column). For illustrative purposes, the alignment of catalytic particles on the $\mathrm{Pd} @ \mathrm{C}$ reference catalyst is also shown schematically (fourth column). 




Figure 10 Evolution of the anodic methanol oxidation peak charge of a Pd@C reference catalyst and Pd-dotted Ni nanowire networks in $0.1 \mathrm{M} \mathrm{NaOH}$ solution containing $0.25 \mathrm{M}$ methanol over 1000 cycles at a scan rate of $100 \mathrm{mV} \mathrm{s}^{-1}$.

be caused by the enormous change in catalyst surface and morphology, probably accompanied by the agglomeration of Pd nanoparticles. Also, methanol evaporation during measurement or electrolyte depletion in close vicinity of the electrode remains possible.

For the dotted structure, no changes in the overall morphology were observed and also, Pd particle agglomeration is strongly suppressed due to the embedding of the particles in the $\mathrm{Ni}$ nanowire surface [40]. Thus, methanol evaporation, electrolyte depletion, and a surface modification of the structures on the atomic scale remain as explanations for any changes in measured performance.
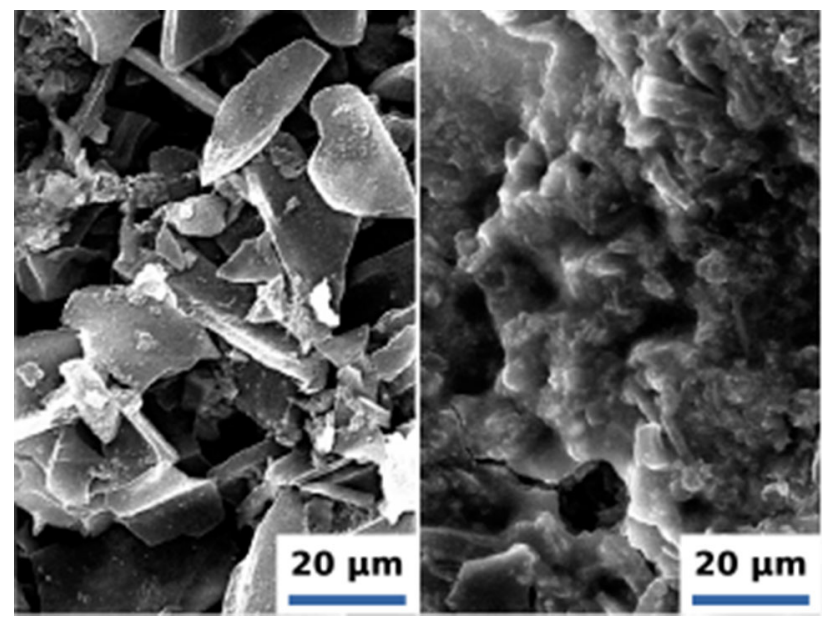

Figure 11 SEM images of Pd@C reference catalyst before (left) and after (right) 1000 cycles in $0.1 \mathrm{M} \mathrm{NaOH}$ and $0.25 \mathrm{M}$ methanol.

\section{Conclusions}

In this work, a new synthesis route to fabricate supported catalyst structures with very little consumption of the noble and thus expensive catalytically active material is introduced. With the combination of surface-selective nanoparticle reduction and electrodeposition, it was possible to create a network of $\mathrm{Ni}$ nanowires decorated with $\mathrm{Pd}$ nuclei, which are embedded in the outer nanowire surface. The amount of noble metal in the final network was determined using ICP-OES and it was shown that this amount can be varied by altering the number of activation steps. Three different Pd loadings were tested in half-cell methanol oxidation measurements. The obtained results indicate that the dotted catalyst structures yields current densities lower than those of a commercial Pd@C reference catalyst. However, all presented dotted structures proved to be more tolerant to high alcohol concentrations than the commercial catalyst. Also, in long-term cycling measurements the dotted catalyst samples were less prone to degradation than the reference catalyst as proven both by the recorded currents and SEM images. ICP-OES analysis of the used electrolytes after long-term cycling indicates, that the dotted nanowire catalysts neither suffer from substrate corrosion nor from $\mathrm{Pd}$ particle dissolution.

\section{Acknowledgements}

T.B. and P.K. acknowledge funding from the German Research Foundation under DFG project No. 342145578. This work was supported by the Helmholtz Association's Initiative and Networking Fund and by the Helmholtz Energy Materials Foundry (HEMF). T.B. would like to thank Torsten Walbert from Technische Universität Darmstadt, Department of Materials and Geoscience for many helpful discussions on this topic and also others.

\section{Funding}

Open Access funding enabled and organized by Projekt DEAL. 


\section{Declarations}

Conflict of interest The authors declare that they have no conflict of interest.

Supplementary Information: The online version contains supplementary material available at http s:/ /doi.org/10.1007/s10853-021-06088-6.

Open Access This article is licensed under a Creative Commons Attribution 4.0 International License, which permits use, sharing, adaptation, distribution and reproduction in any medium or format, as long as you give appropriate credit to the original author(s) and the source, provide a link to the Creative Commons licence, and indicate if changes were made. The images or other third party material in this article are included in the article's Creative Commons licence, unless indicated otherwise in a credit line to the material. If material is not included in the article's Creative Commons licence and your intended use is not permitted by statutory regulation or exceeds the permitted use, you will need to obtain permission directly from the copyright holder. To view a copy of this licence, visit http://creativecommons.org/licen ses/by $/ 4.0 /$.

Supplementary Information: The online version contains supplementary material available at http s://doi.org/10.1007/s10853-021-06088-6.

\section{References}

[1] Andújar JM, Segura F (2009) Fuel cells: History and updating. A walk along two centuries. Renew Sust Energ Rev 13(9):2309-2322

[2] Meier JC, Galeano C, Katsounaros I, Witte J, Bongard HJ, Topalov AA, Baldizzone C, Mezzavilla S, Schüth F, Mayrhofer KJJ (2014) Design criteria for stable Pt/C fuel cell catalysts. Beilstein J Nanotechnol 5:44-67

[3] Hamnett A (1997) Mechanism and electrocatalysis in the direct methanol fuel cell. Catal Today 38(4):445-457

[4] Ganesan A, Narayanasamy M (2019) Ultra-low loading of platinum in proton exchange membrane-based fuel cells: a brief review. Mat Renew Sustain Energy 8(4):18

[5] Antolini E, Perez J (2011) The renaissance of unsupported nanostructured catalysts for low-temperature fuel cells: from the size to the shape of metal nanostructures. J Mater Sci 46(13):4435-4457
[6] Koenigsmann C, Wong SS (2011) One-dimensional noble metal electrocatalysts: a promising structural paradigm for direct methanol fuel cells. Energy Environ Sci 4(4):1161-1176 3

[7] Brouzgou A, Podias A, Tsiakaras P (2013) PEMFCs and AEMFCs directly fed with ethanol: a current status comparative review. J Appl Electrochem 43(2):119-136

[8] Cohen JL, Volpe DJ, Abruna HD (2007) Electrochemical determination of activation energies for methanol oxidation on polycrystalline platinum in acidic and alkaline electrolytes. Phys Chem Chem Phys 9(1):49-77

[9] Møller KT, Jensen TR, Akiba E, Li H-W (2017) Hydrogen A sustainable energy carrier. Prog Nat Sci Mater 27(1):34-40

[10] Antolini E (2009) Palladium in fuel cell catalysis. Energy Environ Sci 2(9):915-931

[11] An L, Zhao TS (2017) Transport phenomena in alkaline direct ethanol fuel cells for sustainable energy production. J Power Sources 341:199-211

[12] Akhairi MAF, Kamarudin SK (2016) Catalysts in direct ethanol fuel cell (DEFC): An overview. Int J Hydrogen Energy 41(7):4214-4228

[13] dos Reis RGCS, Colmati F (2016) Electrochemical alcohol oxidation: a comparative study of the behavior of methanol, ethanol, propanol, and butanol on carbon-supported PtSn, $\mathrm{PtCu}$, and $\mathrm{Pt}$ nanoparticles. J Solid State Electrochem 20(9):2559-2567

[14] Beyhan S, Coutanceau C, Léger JM, Napporn TW, Kadirgan F (2013) Promising anode candidates for direct ethanol fuel cell: Carbon supported PtSn-based trimetallic catalysts prepared by Bönnemann method. Int $\mathrm{J}$ Hydrogen Energy 38(16):6830-6841

[15] Tinoco-Muñoz CV, Reyes-Rodríguez JL, Bahena-Uribe D, Leyva MA, Cabañas-Moreno JG, Solorza-Feria O (2016) Preparation, characterization and electrochemical evaluation of Ni-Pd and Ni-Pd-Pt nanoparticles for the oxygen reduction reaction. Int J Hydrogen Energy 41(48):23272-23280

[16] Xu C, Cheng L, Shen P, Liu Y (2007) Methanol and ethanol electrooxidation on Pt and Pd supported on carbon microspheres in alkaline media. Electrochem Commun 9(5):997-1001

[17] Carvalho LL, Colmati F, Tanaka AA (2017) Nickel-palladium electrocatalysts for methanol, ethanol, and glycerol oxidation reactions. Int $\mathrm{J}$ Hydrogen Energy 42(25):16118-16126

[18] Ye K, Ma X, Cang R, Wang G, Cheng K, Wang G, Cao D (2017) Nickel nanowires decorated with ultra-low palladium loading as an effective electrocatalyst for $\mathrm{NaBH} 4$ oxidation. Catal Sci Technol 7(10):1991-1995 
[19] Obradović MD, Stančić ZM, Lačnjevac UČ, Radmilović VV, Gavrilović-Wohlmuther A, Radmilović VR, Gojković SLj (2016) Electrochemical oxidation of ethanol on palladium-nickel nanocatalyst in alkaline media. Appl Catal B 189:110-118

[20] Guo F, Li Y, Fan B, Liu Y, Lu L, Lei Y (2018) Carbon- and Binder-Free Core-Shell Nanowire Arrays for Efficient Ethanol Electro-Oxidation in Alkaline Medium. ACS Appl Mater Interfaces 10(5):4705-4714

[21] Muench F (2018) Metal Nanotube/Nanowire-Based Unsupported Network Electrocatalysts. Catalysts 8(12):597

[22] Şen F, Gökağaç G (2007) Different Sized Platinum Nanoparticles Supported on Carbon: An XPS Study on These Methanol Oxidation Catalysts. J Phys Chem C 111(15):5715-5720

[23] Wang W, Tian M, Abdulagatov A, George SM, Lee Y-C, Yang R (2012) Three-dimensional Ni/TiO2 Nanowire Network for High Areal Capacity Lithium Ion Microbattery Applications. Nano Lett 12(2):655-660

[24] Walbert T, Antoni M, Muench F, Späth T, Ensinger W (2018) Electroless Synthesis of Highly Stable and FreeStanding Porous Pt Nanotube Networks and Their Application in Methanol Oxidation. ChemElectroChem 5(7):1087-1097

[25] Hasan M, Newcomb SB, Rohan JF, Razeeb KM (2012) Ni nanowire supported 3D flower-like Pd nanostructures as an efficient electrocatalyst for electrooxidation of ethanol in alkaline media. J Power Sources 218:148-156

[26] Muench F, Bohn S, Rauber M, Seidl T, Radetinac A, Kunz U, Lauterbach S, Kleebe H-J, Trautmann C, Ensinger W (2014) Polycarbonate activation for electroless plating by dimethylaminoborane absorption and subsequent nanoparticle deposition. Appl Phys A 116(1):287-294

[27] Gamry Instruments, Inc. Understanding iR compensation application note, 2018

[28] Oelßner W, Berthold F, Guth U (2006) The iR drop - wellknown but often underestimated in electrochemical polarization measurements and corrosion testing. Mater Corros 57(6):455-466

[29] Muench F, Eils A, Toimil-Molares ME, Hossain UH, Radetinac A, Stegmann C, Kunz U, Lauterbach S, Kleebe H-J, Ensinger W (2014) Polymer activation by reducing agent absorption as a flexible tool for the creation of metal films and nanostructures by electroless plating. Surf Coat Technol 242:100-108

[30] Kobayashi Y, Salgueiriño-Maceira V, Liz-Marzán LM (2001) Deposition of Silver Nanoparticles on Silica Spheres by Pretreatment Steps in Electroless Plating. Chem Mater 13(5):1630-1633
[31] Dadvand N, Sohrabi M (1997) Effects of pre-soaking on characteristics of lexan polycarbonate. Appl Radiat Isot 48(8):1127-1131

[32] Erikson H, Sarapuu A, Tammeveski K (2019) Oxygen Reduction Reaction on Silver Catalysts in Alkaline Media: a Minireview. ChemElectroChem 6(1):73-86

[33] Liang ZX, Zhao TS, Xu JB, Zhu LD (2009) Mechanism study of the ethanol oxidation reaction on palladium in alkaline media. Electrochim Acta 54(8):2203-2208

[34] Yang Y-Y, Ren J, Zhang H-X, Zhou Z-Y, Sun S-G, Cai W-B (2013) Infrared Spectroelectrochemical Study of Dissociation and Oxidation of Methanol at a Palladium Electrode in Alkaline Solution. Langmuir 29(5):1709-1716

[35] Barbosa AFB, Oliveira VL, van Drunen J, Tremiliosi-Filho G (2015) Ethanol electro-oxidation reaction using a polycrystalline nickel electrode in alkaline media: Temperature influence and reaction mechanism. J Electroanal Chem 746:31-38

[36] Muench F, Oezaslan M, Rauber M, Kaserer S, Fuchs A, Mankel E, Brötz J, Strasser P, Roth C, Ensinger W (2013) Electroless synthesis of nanostructured nickel and nickelboron tubes and their performance as unsupported ethanol electrooxidation catalysts. J Power Sources 222:243-252

[37] Hu G, Nitze F, Barzegar HR, Sharifi T, Mikołajczuk A, Tai C-W, Borodzinski A, Wågberg T (2012) Palladium nanocrystals supported on helical carbon nanofibers for highly efficient electro-oxidation of formic acid, methanol and ethanol in alkaline electrolytes. J Power Sources 209:236-242

[38] Wang M, Zhang W, Wang J, Wexler D, Poynton SD, Slade RCT, Liu H, Winther-Jensen B, Kerr R, Shi D, Chen J (2013) PdNi Hollow Nanoparticles for Improved Electrocatalytic Oxygen Reduction in Alkaline Environments. ACS Appl Mater Interfaces 5(23):12708-12715

[39] Huang W, Ma X-Y, Wang H, Feng R, Zhou J, Duchesne PN, Zhang P, Chen F, Han N, Zhao F, Zhou J, Cai W-B, Li Y (2017) Promoting Effect of $\mathrm{Ni}(\mathrm{OH}) 2$ on Palladium Nanocrystals Leads to Greatly Improved Operation Durability for Electrocatalytic Ethanol Oxidation in Alkaline Solution. Adv Mater 29(37):1703057

[40] Zhang J, Gao M-R, Luo J-L (2020) In Situ Exsolved Metal Nanoparticles: A Smart Approach for Optimization of Catalysts. Chem Mater 32(13):5424-5441

Publisher's Note Springer Nature remains neutral with regard to jurisdictional claims in published maps and institutional affiliations. 